\title{
Tunneling Charge Transport in Graphene-Based Superconductor Junctions
}

\author{
R.J. WojCiechowski*, L. Kowalewski and M. Nogala \\ Solid State Theory Division, Faculty of Physics, Adam Mickiewicz University \\ Umultowska 85, 61-614 Poznań, Poland
}

We study the spin polarized electron and hole tunneling transport through a graphene-based ferromagnet $\left(G_{1}\right)$-insulator $\left(G_{1}\right)$-superconductor $\left(G_{S}\right)$-insulator $\left(G_{2}\right)$-ferromagnet $\left(G_{2}\right)$ junction. Proximity induced spin polarization and superconductivity in a graphene sheet are assumed to be created by superconducting and ferromagnetic electrodes placed on the top of the graphene. Using a four-dimensional version of the Dirac-Bogoliubov-de Gennes equation with appropriate boundary conditions we investigate the tunneling processes through the junctions. In particular, we present calculations of the amplitudes of normal and Andreev reflections as a function of the energy of the incident electron for a wide range of the model parameters, such as the strength and orientation of the exchange field, the barrier strength, and the distance between the two ferromagnetic layers. The tunneling transport processes in the graphene-based double junction GF/GI/Gs/ GI/GF are compared with those in non-graphene-based junctions.

PACS: $74.45 .+\mathrm{c}, 72.80 . \mathrm{Vp}, 72.25 .-\mathrm{b}$

\section{Introduction}

During the last few years, graphene systems have received considerable theoretical and experimental attention for their intriguing properties and possible device applications. In graphene, the Fermi level is located between two symmetrical conical bands which touch the Fermi energy at six discrete points called the Dirac points located at the edges of the hexagonal Brillouin zone. This implies the zero excitation energy near these points. It was shown [1] that graphene has a linear Dirac-type energy spectrum near each six Dirac points. The linear dispersion is valid up to $1 \mathrm{eV}$ around the Dirac points, which was recently observed in angle-resolved photoemission spectroscopy experiment [2]. This peculiar low-energy electronic structure implies that charge carriers in graphene can be treated like massless relativistic particles. It leads to many unusual phenomena such as the anomalous quantum Hall effect [3] or Klein paradox [4]. When epitaxially grown on a substrate, the graphene systems exhibit new properties, such as ferromagnetism, semiconductivity or superconductivity, which are determined by the proximity-like interactions with the substrate. Unlike a normal metal, in graphene the energy of injected electron can be easily and efficiently controlled by a gate voltage, which is very useful in studies of the charge transport through graphene-based junctions. It was also shown that by changing the Fermi energy $E_{\mathrm{F}}$ via the gate voltage, graphene-based superconductor junctions can exhibit unique local and non-local specular Andreev reflections [5]. This new type of reflection takes place in the weakly doped graphene, i.e. when $E_{\mathrm{F}} \ll \Delta$ ( $\Delta$ - the superconducting energy gap). In this article, we analyse charge transport properties of a graphene-based structure in which two ferromagnet or

* corresponding author; e-mail: wojrysz@amu.edu.pl normal metals are separated from the superconducting layer by two layers of insulating materials. We consider both parallel and antiparallel alignment of magnetization of the ferromagnetic metals. In particular, we calculate the probabilities of the local and non-local Andreev reflections as well as the transmission probability of the elastic cotunneling. In the following we use the formalism of Ref. [6] that was generalized to the spin dependent quantum transport in non-graphene $[7,8]$ and graphene based (e.g. Refs. [9, 10]) multiterminal junctions.

\section{Model and calculations}

We consider single particle transport in a graphene-based junction $\mathrm{GM}_{1} / \mathrm{GI}_{1} / \mathrm{G}_{\mathrm{S}} / \mathrm{GI}_{2} / \mathrm{GM}_{2}$, consisting of two metallic graphene $\left(\mathrm{M}_{i}, i=1,2\right)$ (normal or ferromagnetic) electrodes and a superconducting graphene layer $(\mathrm{S})$ separated from the electrodes by thin insulating graphene interfaces $\left(\mathrm{GI}_{i}, i=1,2\right)$. The ferromagnetism and superconductivity in graphene are induced due to the proximity effects by the ferromagnetic and superconducting electrodes, respectively, deposited on the top of the junction. In this paper we have analysed the behaviour of the different scattering probabilities. In particular, we have concentrated on the dependence of the local (AR) and non-local (CAR) Andreev reflections, and the elastic cotunneling (ET) on the thickness $L$ of the superconducting layer. The values of the thickness should be compared with the superconducting coherence length given by $\xi=h v_{\mathrm{F}} / \pi \Delta$. A typical $s$-wave conventional superconductor like aluminum has $\xi=1600 \mathrm{~nm}$, and similar values are asummed for the proximity induced superconductor in graphene.

In our analysis we use the Dirac-Bogolyubov-de Gennes (DBdG) equations [1] in the following form:

$$
\left(\begin{array}{cc}
H_{\alpha \sigma}-E_{\mathrm{F}} \hat{1} & \Delta(\boldsymbol{r}) \\
\Delta^{\dagger}(\boldsymbol{r}) & -\left[H_{\alpha \sigma}-E_{\mathrm{F}} \hat{1}\right]
\end{array}\right) \Psi_{\alpha}=E \Psi_{\alpha},
$$


where $\Psi_{\alpha}=\left(\Psi_{\mathrm{A} \alpha \sigma}, \Psi_{\mathrm{B} \alpha \sigma}, \Psi_{\mathrm{A} \alpha^{\prime} \sigma}^{*}, \Psi_{\mathrm{B} \alpha^{\prime} \sigma}^{*}\right)^{\mathrm{T}}$ stands for four-component wave function and $\mathrm{T}$ is the transpose. The subscripts have the following meaning: A and B denote the two sublattices of the graphene lattice, $\alpha\left(\alpha^{\prime}\right)$ indicates the valley $K\left(K^{\prime}\right)$ in the Brillouin zone. Index $\sigma=1(\uparrow)$ denotes the spin-up electron and the spin-down hole, whereas $\sigma=-1(\downarrow)$ labels the spin-down electron and the spin-up hole. The quasiparticle energy $E$ is measured from the Fermi energy $E_{\mathrm{F}}$, and around each of the Dirac points, low energy electrons and holes have linear dispersion. Thus, we get the conical-like conduction and valence bands which touch at each the Dirac points. This is the origin of a graphene based gapless semiconductor with a relativistic-like dispersion relation. The single-particle Hamiltonian $H_{\alpha \sigma}$ for $K$ valley has the form $H_{\alpha \sigma}=-\mathrm{i} \hbar v_{\mathrm{F}}\left(\sigma_{x} \partial_{x}+\sigma_{y} \partial_{y}\right)+U(x)-\sigma h(x)$, where $U(x)$ is the electrostatic potential which can be adjusted via a gate voltage or doping. We assume $U(x)=0$ for $x<-d$ and $x>L+d$ in the $\mathrm{GM}_{i}(i=1,2)$ regions, $U(x)=V_{i}(i=1,2)$ for $-d<x<0$ and $L<x<L+d$ in insulating regions $\mathrm{GI}_{i}(i=1,2)$, and $U(x)=-U_{0}$ for $0<x<L$ in the superconducting layer GS. For the ferromagnetic electrodes, we adopt the Stoner model with the exchange fields $h(x)=h \theta(-x-d)+h_{\mathrm{R}} \theta(x-L-d)$, and $h_{\mathrm{R}}=h$ in the case of the parallel configuration of the ferromagnet electrodes, and $h_{\mathrm{R}}=-h$ for the antiparallel alignment.

The wave function, describing the quasiparticle propagation across the junction, can be obtained from extended DBdG equations. The solution of Eq. (1) for all regions of the junctions and for the injection of an electron with spin $\sigma$ from the left/right $(+/-)$ ferromagnetic electrode, with the energy $E$, and the angle of incidence $\Theta$ can be written in the following form:

in the ferromagnetic regions $(x<-d$ or $x>L+d)$ :

$$
\begin{aligned}
& \Psi_{ \pm}^{\mathrm{e}}(x)=\left[1, \pm \mathrm{e}^{ \pm \mathrm{i} \Theta}, 0,0\right]^{\mathrm{T}} \mathrm{e}^{ \pm \mathrm{i} p_{\mathrm{e} \sigma} \cos \Theta x}, \\
& \Psi_{ \pm}^{\mathrm{h}}(x)=\left[0,0,1, \mp \mathrm{e}^{ \pm \mathrm{i} \Theta_{\AA}^{\sigma}}\right]^{\mathrm{T}} \mathrm{e}^{ \pm i p_{\mathrm{h} \sigma} \cos \Theta_{\AA}^{\sigma} x},
\end{aligned}
$$

in the insulating regions $(-d<x<0$ for $i=1$ or $L<x<L+d$ for $i=2)$ :

$$
\begin{aligned}
& \Psi_{i l \pm}^{\mathrm{h}}(x)=\left[0,0,1, \mp \mathrm{e}^{ \pm \mathrm{i} \Theta_{i 0}^{\sigma \mathrm{A}}}\right]^{\mathrm{T}} \mathrm{e}^{ \pm \mathrm{i} p_{\mathrm{h} i} \cos \Theta_{i 0}^{\sigma \mathrm{A}} x}, \\
& \Psi_{i l \pm}^{\mathrm{e}}(x)=\left[1, \pm \mathrm{e}^{ \pm \mathrm{i} \Theta_{i 0}^{\sigma}}, 0,0\right]^{\mathrm{T}} \mathrm{e}^{ \pm \mathrm{i} p_{\mathrm{e} i} \cos \Theta_{i 0}^{\sigma} x}
\end{aligned}
$$

in the superconducting region $(0<x<L)$ :

$$
\begin{aligned}
& \Psi_{S \pm}^{\mathrm{e}}(0<x<L)=\left[u, u \mathrm{e}^{\mathrm{i} \Theta_{ \pm}^{\sigma}}, v, v \mathrm{e}^{\mathrm{i} \Theta_{ \pm}^{\sigma}}\right]^{\mathrm{T}} \\
& \quad \times \mathrm{e}^{\mathrm{i} q^{\mathrm{e}} \cos \Theta_{ \pm}^{\sigma} x}, \\
& \Psi_{S \pm}^{\mathrm{h}}(0<x<L)=\left[v, v \mathrm{e}^{\mathrm{i} \Theta_{ \pm}^{\sigma}}, u, u \mathrm{e}^{\mathrm{i} \Theta_{ \pm}^{\sigma}}\right]^{\mathrm{T}} \\
& \quad \times \mathrm{e}^{\mathrm{i} q^{\mathrm{h}} \cos \Theta_{ \pm}^{\sigma} x},
\end{aligned}
$$

where $p_{\mathrm{e} \sigma}=\left(E+E_{\mathrm{F}}+\sigma h\right), p_{\mathrm{h} \sigma}=\left(E-E_{\mathrm{F}}+\sigma h\right)$, $p_{\mathrm{e} i}=\left(E+E_{\mathrm{F}}-V_{i}\right), p_{\mathrm{h} i}=\left(E-E_{\mathrm{F}}+V_{i}\right) ;(i=1,2)$, $q^{\mathrm{e}}=\left(E_{\mathrm{F}}+U_{0}+\sqrt{E^{2}-\Delta^{2}}\right), q^{\mathrm{h}}=\left(E_{\mathrm{F}}+U_{0}-\sqrt{E^{2}-\Delta^{2}}\right)$, and $u$ and $v$ are the BCS coherence factors. The angles $\Theta_{\mathrm{A}}^{\sigma}, \Theta_{i 0}^{\sigma}, \Theta_{i 0}^{\sigma \mathrm{A}}, \Theta_{ \pm}^{\sigma}$ are related to the angle of incidence $\Theta$ and can be determined from the assumed momentum conservation in the $y$ direction, i.e. where the system is translationally invariant. From the conservation of momentum, we find critical angles of incidence $\alpha_{\sigma}^{i}(i=1,2$, $3,4,5)$. For greater angles the waves functions describing the reflection processes become evanescent, and thus, these processes do not contribute to any charge transport. The critical angle is defined as $\min \left[\alpha_{\sigma}^{i}\right]$ and $\alpha_{\sigma}^{i}$ are defined as follows:

$$
\begin{aligned}
& \alpha_{\sigma}^{1}=\arcsin \left(\left|E+\sigma h-E_{\mathrm{F}}\right| D^{-1}\right), \\
& \alpha_{\sigma}^{j}=\arcsin \left(|E-| E_{\mathrm{F}}-V_{j}|| D^{-1}\right), \quad j=2,3, \\
& \alpha_{\sigma}^{4}=\arcsin \left(\left|E_{\mathrm{F}}+U_{0}\right| D^{-1}\right), \\
& \alpha_{\sigma}^{5}=\arcsin \left(\left|E+\sigma^{\mathrm{R}} h-E_{\mathrm{F}}\right| D^{-1}\right),
\end{aligned}
$$

where $D=\left|E+\sigma h+E_{\mathrm{F}}\right|$ and $\sigma^{\mathrm{R}}= \pm 1$ stands for the spin value in the right graphene layer. The probability amplitudes, for the AR, CAR and ET processes in graphene-based junctions, are determined from the appropriate boundary conditions, imposing only continuity of the wave functions.

The appropriate transport coefficients for a right moving spin up electron are determined by imposing the boundary conditions that match the following wave functions at all the interfaces:

$$
\begin{aligned}
& \Psi_{\text {left }}(x)=\Psi_{+}^{\mathrm{e}}(x)+b \Psi_{-}^{\mathrm{e}}(x)+a_{\mathrm{AR}} \Psi_{-}^{\mathrm{h}}(x), \\
& \Psi_{i I}(x)=p_{i} \Psi_{i I+}^{\mathrm{e}}(x)+q_{i} \Psi_{i I-}^{\mathrm{e}}(x)+m_{i} \Psi_{i I+}^{\mathrm{h}}(x) \\
& \quad+n_{i} \Psi_{i I-}^{\mathrm{h}}(x), \\
& (i=1,2) \\
& \Psi_{S}(x)=p_{S} \Psi_{S+}^{\mathrm{e}}(x)+q_{S} \Psi_{S-}^{\mathrm{e}}(x)+m_{S} \Psi_{S+}^{\mathrm{h}}(x) \\
& \quad+n_{S} \Psi_{S-}^{\mathrm{h}}(x), \\
& \Psi_{\text {right }}(x)=c_{\mathrm{ET}} \Psi_{+}^{\mathrm{eR}}(x)+a_{\mathrm{CAR}} \Psi_{+}^{\mathrm{hR}}(x),
\end{aligned}
$$

where $b_{\mathrm{NR}}, a_{\mathrm{AR}}, a_{\mathrm{CAR}}$, and $c_{\mathrm{ET}}$ stand for the amplitudes of normal, local Andreev, non-local Andreev reflections, and elastic cotunneling, respectively.

The remaining coefficients in Eq. (12) are responsible for transmission processes in insulating and superconducting layers. The boundary conditions lead to a system of 16 linear equations whose solutions give all probability amplitudes defined in Eq. (12). The probability current conservation requires that the probabilities of outgoing processes satisfy the normalization condition.

\section{Conclusions}

In summary, we have considered the charge transport through the graphene based multiterminal junctions with the gate voltages $\left(V_{1}, V_{2}\right)$ applied to the insulator as well to the superconductor $(U)$ layers. In Fig. 1 we have presented how the probabilities of ET, CAR and AR depend on the magnetic configurations of the ferromagnetic electrodes. For parallel alignment (see Fig. 1a) ET dominates 
wheras for antiparallel one (see Fig. 1b) we have observed the dominance of CAR, however for larger values of $L$ $(L>\xi) \mathrm{AR}$ is greater than CAR.

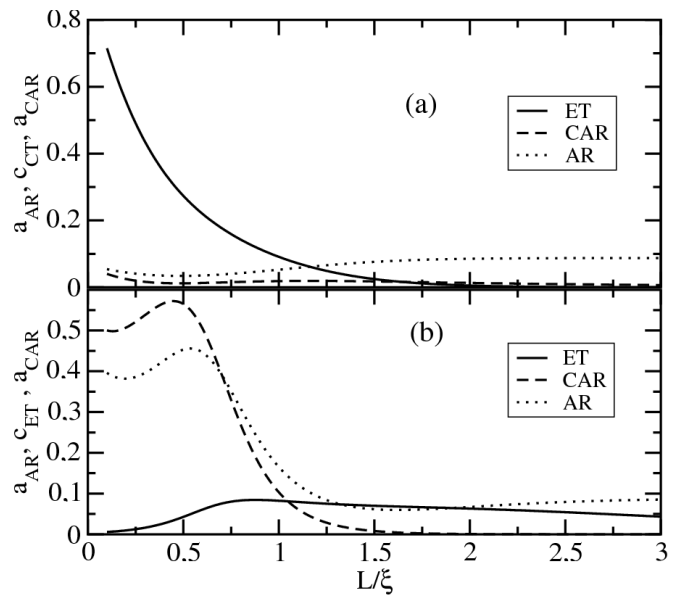

Fig. 1. Probabilities of the elastic cotunneling $\left(c_{\mathrm{ET}}\right)$, crossed Andreev reflection $\left(a_{\mathrm{CAR}}\right)$, and local Andreev reflection $\left(a_{\mathrm{AR}}\right)$ as a function of the width of the superconducting layer $L$ relative to the superconducing coherence length $\xi$, for the following parameters: $h / \Delta=0.8$, $\theta=0.8, d / \xi=0.1, E / \Delta=0.50, V_{1} / \Delta=0.1$, $V_{2} / \Delta=1.1, U_{0} / \Delta=0.1$; (a) and (b) stand for parallel and antiparallel configurations, respectively.

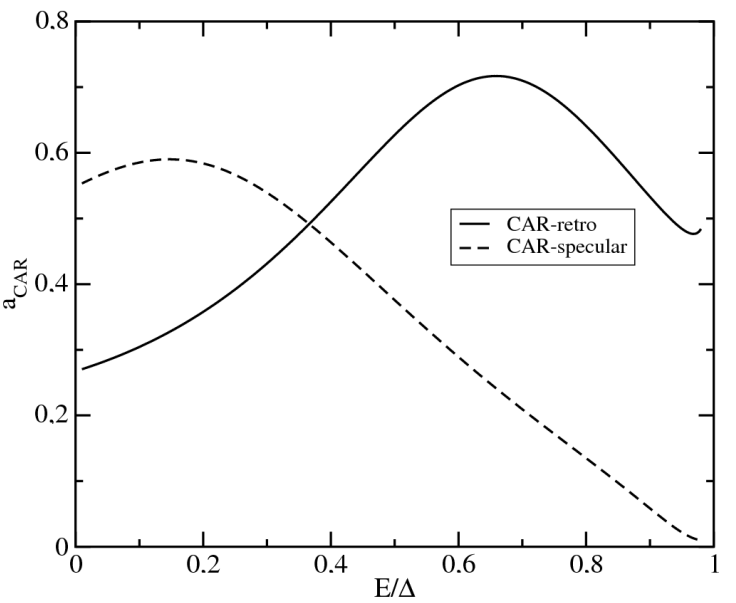

Fig. 2. Probabilities of the specular $\left(E_{\mathrm{F}} / \Delta=0.02\right)$ and retro $\left(E_{\mathrm{F}} / \Delta=100\right)$ crossed Andreev reflections as a function of energy $E$, for the following parameters: $h / \Delta=0.3, \theta=0.2, d / \xi=1.6, V_{1} / \Delta=0.6, V_{2} / \Delta=0.2$, $U_{0} / \Delta=0.1$.

In Fig. 2 we have plotted the probabilities of the specular and retro non-local Andreev reflections as function of energy. The specular Andreev reflections takes place in the weakly doped graphene i.e. if $E_{\mathrm{F}} \ll \Delta$. In the heavily doped graphene i.e. if $E_{\mathrm{F}} \gg \Delta$, the retro Andreev reflection appears. For $E / \Delta \leq 1$ the retro reflection dominates if $E_{\mathrm{F}} \gg \Delta$, while the specular reflection dominates if $E_{\mathrm{F}} \ll \Delta$ [5]. In Fig. 2, we can see that for very small values of energy the specular reflection can dominate. However, it is hardly a universal feature but merely a consequence of the choice of the model parameters. The interesting fact is that for the ferromagnetic graphene-based junctions, separated by two insulator layers, the specular Andreev reflection, unlike the retro Andreev reflection, is very sensitive to the change of the polarizations of the ferromagnetic electrodes. The retro Andreev reflection oscillates with the width of insulators layers $d$ and the gate voltages $V_{1}$ and $V_{2}$ applied to the insulators, regardless of the strength of the magnetic field $h$.

It is worth mentioning that the wave functions are propagating in the ferromagnetic regions for all values of the quasiparticle energy, while in the superconducting region only for the energies which are greater than the superconducting gap $\Delta$. For the energies lower than $\Delta$, the wave functions become evanescent along their propagation direction, inside the superconducting region, and they decay exponentially for $L>\xi$. We have observed that analogously to non-graphene double junctions [11], the probabilities of the coherent single particle charge transport processes (AR, CAR, and ET) oscillate with the energy (for $E>\Delta$ ) and the thickness $L$ of the superconducting layer and vanish for $E<\Delta$ if $L \gg \xi$. The oscillations, with a period of geometrical resonance, are due to interference of incoming and outgoing charge carriers. In the limit $L \gg \xi$ the sub-gap transmission contains only the local Andreev process. For $L \approx \xi$ the electron elastic cotunneling begins to play a role and a further decrease in $L$ activates also the non-local Andreev reflection.

\section{References}

[1] P.R. Wallace, Phys. Rev. 71, 622 (1947).

[2] S.Y. Zhou, G.H. Gweon, J, Graf, A.V. Fedorov, C.D. Spataru R.D. Diehl, Y. Kopelevich, D.-H. Lee, S.G. Louie, A. Lanzara, Nat. Phys. 2, 595 (2006).

[3] K.S Novoselov, A.K. Geim, S.V. Morozov, D. Jing, M.I. Katsnelson, I.V. Grigorieva, S.V. Dubonos, A.A. Firsov, Nature (London) 438, 197 (2005).

[4] B. Huard, J.A. Sulpizio, N. Stander, K. Todd, B. Yang, D. Goldhaber-Gordon, Phys. Rev. Lett. 102, 026807 (2009).

[5] C.W.J. Beenakker, Phys. Rev. Lett. 97, 067007 (2006).

[6] G.E. Blonder, M. Tinkham, T.M. Klapwijk, Phys. Rev. B 25, 4515 (1982).

[7] S. Takahashi, H. Imamura, S. Maekawa, Phys. Rev. Lett. 82, 3911 (1999).

[8] R.J. Wojciechowski, L. Kowalewski, Acta Phys. Pol. A 118, 249 (2010).

[9] C. Benjamin, J.K Pachos, Phys. Rev. B 78, 235403 (2008).

[10] M. Salehi, G. Rashedi, Physica C 470, 703 (2010).

[11] M. Božević, Z. Radović, Phys. Rev. B 66, 134524 (2002). 